\title{
KCC2-GABAA pathway correlates with the analgesic effect of electro-acupuncture in CCI rats
}

\author{
SI-SI LI ${ }^{1}$, WEN-ZHAN TU ${ }^{1,2}$, CHENG-QIAN JIA ${ }^{1}$, XIA JIANG $^{1}$, XIN-RU QIAN ${ }^{2,3}$, GUAN-HU YANG ${ }^{2,4}$, \\ QI-MIAO HU ${ }^{1}$, WEN-CI CHEN ${ }^{1}$, BIN LU ${ }^{5}$ and SONG-HE JIANG ${ }^{1,2}$ \\ ${ }^{1}$ Department of Physical Medicine and Rehabilitation, The Second Affiliated Hospital and Yuying Children's Hospital of \\ Wenzhou Medical University; ${ }^{2}$ Integrative and Optimized Medicine Research Center, \\ China-US Institute for Acupuncture and Rehabilitation of Wenzhou Medical University, Wenzhou, Zhejiang 325027, \\ P.R. China; ${ }^{3}$ Department of Rehabilitation, NYU Langone Medical Center, New York, NY 10016; \\ ${ }^{4}$ Department of Specialty Medicine, Ohio University, Athens, OH 45701, USA; ${ }^{5}$ Protein Quality Control and \\ Diseases Laboratory, Attardi Institute of Mitochondrial Biomedicine, School of Life Sciences, \\ Wenzhou Medical University, Wenzhou, Zhejiang 325035, P.R. China
}

Received August 16, 2016; Accepted May 8, 2017

DOI: $10.3892 / \mathrm{mmr} .2018 .8766$

\begin{abstract}
Potassium-chloride cotransporter 2 (KCC2) has been indicated to serve a crucial role during chronic neuropathic pain (NP). Following the emergence of NP, $\gamma$-aminobutyric acid (GABA) A receptor-mediated signaling may be further impaired by the changes of KCC2 chloride anion gradient. In the present study, the authors investigate the effect of electro-acupuncture (EA) on the behavior and the expression of KCC2 and GABAA receptor $\gamma 2$ subunit in the spinal cord of chronic constriction injury (CCI) model rats. A total of 60 adult male Sprague-Dawley rats were divided into four groups: Normal group, sham-CCI group, CCI group and CCI+EA group. The effect of EA was assessed via the values of mechanical withdrawal threshold and thermal withdrawal latency, which were significantly improved upon stimulation of the ST-36 and GB-34 acupoints. In addition, a marked reduction in both the mRNA and protein levels of $\mathrm{KCC} 2$ and GABAA receptor $\gamma 2$ subunit was observed in the spinal cord following loose ligation of the sciatic nerve. The reductions
\end{abstract}

Correspondence to: Professor Song-He Jiang, Department of Physical Medicine and Rehabilitation, The Second Affiliated Hospital and Yuying Children's Hospital of Wenzhou Medical University, 268 Xueyuanxi Road, Wenzhou, Zhejiang 325027, P.R. China

E-mail: songhe.jiang@gmail.com

Professor Bin-Lu, Protein Quality Control and Diseases Laboratory, Attardi Institute of Mitochondrial Biomedicine, School of Life Sciences, Wenzhou Medical University, University Town, Chashan, Wenzhou, Zhejiang 325035, P.R. China

E-mail: lubmito@wmu.edu.cn

Key words: electro-acupuncture, KCC2, GABAA receptor, chronic constriction injury, analgesic in $\mathrm{KCC} 2$ and GABAA receptor $\gamma 2$ subunit expression were reversed by EA treatment. These results support the notion that KCC2 and GABAA receptor $\gamma 2$ subunit contribute to NP following peripheral nerve injury and extend the understanding of the analgesic effects of EA on NP.

\section{Introduction}

Neuropathic pain (NP) is defined as a chronic pain state caused by damage or abnormal function of the peripheral or central nervous system (CNS) $(1,2)$. This pain may result from a wide variety of pathological processes including inflammation, nerve trauma, nerve compression, genetic abnormalities and metabolic disorders (3). Previous studies indicate that the expression of potassium-chloride cotransporter $2(\mathrm{KCC} 2)$ is decreased in models of inflammation pain and NP (4,5). Coull et al (6) first proposed that KCC2 has a role in the production and development of NP. Two isoforms of the cation- $\mathrm{Cl}(-)$ cotransporter family are expressed in neurons and modulate neurotransmission, the KCC and sodium potassium-chloride-co transporters (NKCC), mediate the electroneutral movements of $\mathrm{K}+$ and $\mathrm{Cl}$ - ions across cell membranes in a coupled manner (7). KCC2 involves in the control of numerous neuronal processes, including migration, dendritic outgrowth, the formation of synaptic connections, and exerts a great influence on the establishment of neuronal [Cl-]i $(6,8)$. It maintains a low concentration of intracellular $\mathrm{Cl}$-, which is essential for inhibitory synaptic transmission by $\gamma$-aminobutyric acid (GABA) in the mature nervous system (9). Previous work has demonstrated that GABA-ergic interneurons are important in spinal nociceptive processing and nociceptive attenuation $(10,11)$. GABAA receptors, which are the major synaptic targets for the neurotransmitter GABA, are located in primary afferent terminals and interneurons in laminae I-IV of the spinal cord dorsal horn $(12,13)$. The GABAA receptors are ligand-gated chloride channels in the CNS and mediating inhibitory neurotransmission, consist of subunits such as $\alpha 1-6$, 
$\beta 1-3, \gamma 1-3, \delta, \varepsilon, \pi, \theta$ and $\rho 1-3(14,15)$, the most common subtype is composed of $\alpha 1, \beta 2$ and $\gamma 2$ subunits (16). The location of $\gamma 2$ subunit mRNA-containing neurons differs from those containing $\alpha$ - or $\beta$-subunits. Earlier work has indicated that the distribution of strongly labeled $\gamma 2$ subunit nuclei partially coincided with that of glutamate decarboxylase, suggesting that the GABAA receptor $\gamma 2$ subunit may be involved in the mechanism of GABA-ergic transmission (17).

Electro-acupuncture (EA) is known to relieve both peripheral NP and acute or chronic inflammatory pain via electrical stimulation $(18,19)$. However, little is understood about its mechanism of action. In the present work, the acupoints Zusanli (ST-36) and Yanglingquan (GB-34) were applied following the production of NP by chronic constriction injury (CCI).

The current study investigated the application of EA on ST-36 and GB-34 acupoints and its effect on modulating mechanical allodynia and thermal hyperalgesia in rats with NP. In addition, the relationship between EA and the KCC2-GABAA receptor signaling pathway in CCI rats was investigated.

\section{Materials and methods}

Experimental animals. Male Sprague-Dawley rats (180-200 g) were purchased from Wenzhou Medical University (Wenzhou, China). All experiments were maintained and cared for according to the guidelines of the National Institutes of Health Guide for the Care and Use of Laboratory Animals (NIH Publications no. 80-23, revised 1978). The experimental protocol was approved by the Institutional Animal Care and Use Committee of Wenzhou Medical University (Wenzhou, China). All efforts were made to minimize the animals' pain and discomfort during the experiments. A total of 60 male Sprague-Dawley rats were housed in a climate-controlled room with a constant temperature $\left(22 \pm 2^{\circ} \mathrm{C}\right)$ and humidity $(55 \pm 5 \%)$ in a 12 -h light/dark cycle with food and water available ad libitum. Rats were randomly divided into four groups: Normal group, sham-CCI group, CCI group and CCI plus EA group. All experiments were conducted with the experimenters blinded to treatment conditions.

CCI surgery. CCI to the sciatic nerve of the right hind limb in rats was performed based on previous description (2). Briefly, animals were anaesthetized with $4 \%$ chloral hydrate $(10 \mathrm{ml} / \mathrm{kg}$; i.p.). The sciatic nerve of the right hind limb was exposed at the middle of the thigh by blunt dissection. To prevent the interruption of blood circulation through the epineural vasculature, four chromic gut ligatures were loosely tied (4.0 silk) around the nerve with spacing at $\sim 1 \mathrm{~mm}$. In the sham-CCI group rats, the right sciatic nerve was exposed for 2-3 min, but was not ligated. Following surgery, the skin was closed with a single suture, and the animals were allowed to recover for 7 days.

Mechanical withdrawal threshold (MWT). Mechanical allodynia and thermal hyperalgesia are reproducible and sensitive behavioral readouts of neuropathic pain. Behavioral testing was conducted prior to surgery and on days 3, 5, 7, 10, 12 and 14 following surgery. Animals were allowed to acclimate to elevated cages $(20 \times 14 \times 16 \mathrm{~cm})$ with a wire mesh bottom. MWT was measured by assessing hind paw sensitivity to innocuous mechanical stimulation. Ascending mechanical pressure (0-70 g) was applied to the plantar aspect of right hind paw by the 2392 Electronic von Frey Anesthesiometer (IITC Life Science, Woodland Hills, CA, USA). Lifting, licking the paw and running away were all considered as positive responses. The maximum applied pressure was recorded. The MWT of each animal was the average of six measurements taken at 5 min intervals.

Thermal withdrawal latency (TWL). In this assay, rats were placed in a transparent, square, bottomless acrylic box $(17 \times 11.5 \times 14 \mathrm{~cm})$ and allowed to adapt for $20 \mathrm{~min}$. Responses to thermal stimulation were evaluated using a 37370 plantar test apparatus (UgoBasile SRL, Milan, Italy) as a source of radiant heat. A beam of focused light set at $60^{\circ} \mathrm{C}$ was directed towards the plantar surface of the hind paw, and the maximum latency time was recorded. The time to purposeful withdrawal of the foot from the beam of light was measured. A cut-off time was set at $40 \mathrm{sec}$ to prevent tissue damage. Every hind paw was tested alternately at $5 \mathrm{~min}$ intervals. The results obtained for each rat were expressed in sec (sec) as the mean of five withdrawal latencies. Finally, the average value was used for statistical analysis.

EA stimulation. EA treatment was given between 9:00 and 11:00 a.m. every day and lasted for 7 days. The rats were maintained in an immobilization apparatus designed by the authors' laboratory (patent application number: 201110021482.5, State Intellectual Property Office) (20-22). During EA treatment, stainless steel needles were percutaneously inserted into the right hind leg at two points: Zusanli acupoint (ST-36, $5 \mathrm{~mm}$ below the head of the fibula under the knee joint, and $2 \mathrm{~mm}$ lateral to the anterior tubercle of the tibia) and the Yanglingquan acupoint (GB-34, 5 $\mathrm{mm}$ superior-lateral to ST-36; Fig. 1) (23,24). It has been previously reported that $2 \mathrm{~Hz}$ induced analgesia mediated by the release of met-enkephalin and $\beta$-endorphin, while $100 \mathrm{~Hz}$ via release of dynorphin- $\mathrm{A}$ in the CNS (25). Combining the low $(2 \mathrm{~Hz})$ and high $(100 \mathrm{~Hz})$ frequencies alternately (named ' $2 / 100 \mathrm{~Hz}$ ') elicits a synergistic analgesic effect (26). The intensity of the stimulation was set at 1.5 mA, which elicited a slight twitch. A HANS-200E (Jisheng Medical Instruments Co., Ltd., Nanjing, China) was used to deliver the acupuncture stimulus and enabled the performance of EA. Acupuncture needles were inserted 2-3 mm deep and stimulated electrically at a frequency of $2 / 100 \mathrm{~Hz}$ to permit the muscle to shrink slightly. The total stimulation period was $30 \mathrm{~min}$.

Immunohistochemistry. Rats ( $\mathrm{n}=5$ per group) were anaesthetized as described above and perfused via the aorta with $0.9 \%$ normal saline, followed by addition of $4 \%$ paraformaldehyde in phosphate-buffered saline on day 14 following surgery. Spinal cord segments (L4-L6) were collected and fixed in $4 \%$ paraformaldehyde for $24 \mathrm{~h}$. Following fixation, they were dehydrated, cleared and embedded in paraffin for transverse paraffin sections. The paraffin sections (5- $\mu \mathrm{m}$ thick) were made and mounted on poly-L-lysine-coated slides for immunohistochemistry. To block the activity of endogenous peroxidase, the slides were incubated in $3 \% \mathrm{H}_{2} \mathrm{O}_{2}$ for $10 \mathrm{~min}$ 
at room temperature and blocked in $10 \%$ normal goat serum with $0.3 \%$ Triton X-100 in PBS for $1 \mathrm{~h}$. Sections were probed with mouse anti-KCC2 (1:200; cat. no. ab134300; Abcam, Cambridge, MA, USA) and rabbit anti-GABAA receptor $\gamma 2$ subunit (1:100; cat. no. orb5291; Biorbyt Ltd., Cambridge, UK) antibodies overnight at $4^{\circ} \mathrm{C}$. The next day, slides were incubated for $30 \mathrm{~min}$ at $37^{\circ} \mathrm{C}$ with the following secondary antibodies: Two-step plus Poly-horseradish peroxidase (HRP) anti-mouse immunoglobulin (Ig)G detection system (1:1; cat. no. PV-6002; Beijing Zhongshan Golden Bridge Biotechnology, Co., Ltd., Beijing, China) and two-step plus Poly-HRP anti-rabbit IgG detection system (1:1; cat. no. PV-6001; Beijing Zhongshan GoldenBridge Biotechnology, Co., Ltd.). Slides were then stained with DAB for $20 \mathrm{sec}$ and counterstained with haematoxylin for $8 \mathrm{~min}$ at room temperature. Finally, images were captured via a light microscope (Olympus Corporation, Tokyo, Japan). Image-Pro Plus 5.1 software (Media Cybernetics, Inc., Rockville, MD, USA) was used to define and determine positive regions of interest.

Western blotting. Rats ( $\mathrm{n}=5$ per group) were deeply anesthetized as described above and perfused with $0.9 \%$ normal saline. The L4-L6 spinal cord segments $(1 \mathrm{~cm})$ were dissected on day 14 post-CCI surgery. The segments were lysed in fresh radioimmunoprecipitation assay protein lysis buffer containing phenylmethylsulfonyl fluoride (PMSF) (RIPA: $\mathrm{PMSF}=100: 1)$ for $30 \mathrm{~min}$ and centrifuged at 15,294 $\mathrm{x} \mathrm{g}$ for $5 \mathrm{~min}$ at $4^{\circ} \mathrm{C}$, and the supernatant was subsequently collected. Protein concentration of each sample was determined using a bicinchoninic acid assay protein assay kit (Beyotime Institute of Biotechnology, Haimen, China). The proteins were mixed with loading buffer, heated to $100^{\circ} \mathrm{C}$ for $10 \mathrm{~min}$ and separated on an 8 or $10 \%$ Tris-HCl SDS-PAGE gel (Bio-Rad Laboratories, Inc., Hercules, CA). Following electrophoresis, the proteins were transferred to a polyvinylidene fluoride membrane. The membranes were blocked with $5 \%$ non-fat milk for $2 \mathrm{~h}$ and incubated overnight at $4^{\circ} \mathrm{C}$ with the following antibodies: Mouse anti-KCC2 (1:400; cat. no. ab134300; Abcam); rabbit anti-GABAA receptor $\gamma 2$ subunit (1:400; cat. no. orb5291; Biorbyt Ltd.). Next, the membranes were incubated with peroxidase-conjugated goat anti-mouse IgG (H+L) (1:5,000; cat. no. BL001A; Biosharp, Hefei, China) or peroxidase-conjugated goat anti-rabbit $\operatorname{IgG}(\mathrm{H}+\mathrm{L})$ secondary antibody (1:5,000; cat. no. BL003A; Biosharp). Following being washed, the labeled proteins were visualized using the enhanced chemiluminescence (ECL) kit (Beyotime Institute of Biotechnology). The quantity of band intensity was detected by aDNR microchemiluminescence gel imaging system (DNR Bio-Imaging Systems, Jerusalem, Israel) and using AlphaEase FC 4.0.0 software from ProteinSimple, (San Jose, CA, USA). The band densities were normalized to GAPDH.

Reverse transcription-quantitative polymerase chain reaction $(R T-q P C R)$. Total RNA was extracted from the L4-L6 segment with TRIzol (Invitrogen; Thermo Fisher Scientific, Inc., Waltham, MA, USA) according to the manufacturer's instructions ( $\mathrm{n}=5$ per group). Total RNA $(1 \mu \mathrm{g})$ was reverse transcribed using High Capacity RNA-to-cDNA Master Mix (cat. no. A3500; Promega Corporation, Madison, WI, USA). Real-time amplification was conducted using SYBR Green

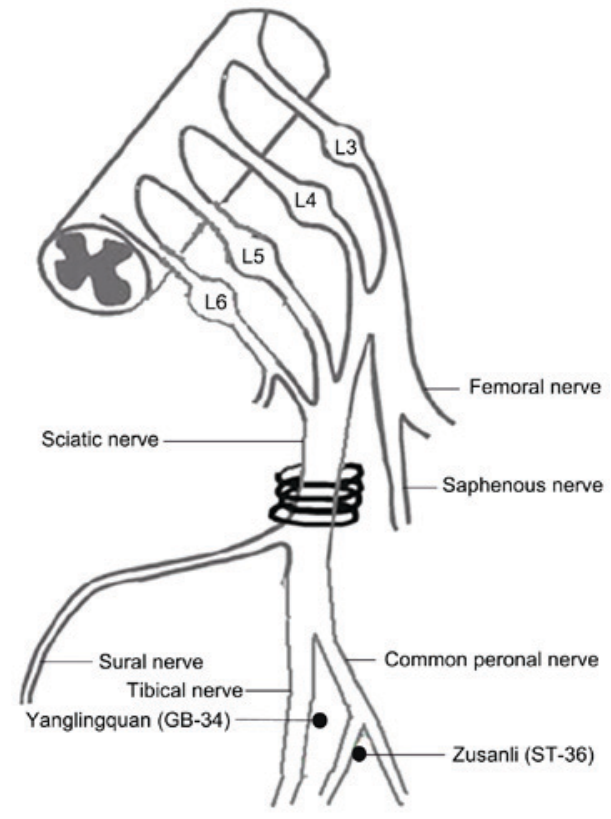

Figure 1. The location of sciatic nerve and the other nerves near to where it is described. The sciatic nerve of the right hind limb was exposed at the middle of the thigh, and four chromic gut ligatures were loosely tied around the nerve with $\sim 1 \mathrm{~mm}$ spacing to prevent the interruption of blood circulation through the epineural vasculature. The two black points represent 'Zusanli' (ST-36) and 'Yanglingquan' (GB-34) acupoints.

Supermix (Toyobo Co., Ltd., Osaka, Japan) and a Light Cycler 480 system (Roche Diagnostics GmbH, Mannheim, Germany). Primer sequences were the following: KCC2 forward, 5'-AGCAAAGAGCACGAAGAAGC-3' and reverse, 5'-GCCGCAGAAAGAGGATAACA-3'; GABAA receptor $\gamma 2$ subunit forward, 5'-TTCTGTCCTGGGTGTCCTTC-3' and reverse, 5'-AGAGACTTCCGGGCTATGGT-3'; RPS16 forward, 5'-AAGTCTTCGGACGCAAGAAA-3' and reverse, 5'-TTGCCCAGAAGCAGAACAG-3'. The PCR reactions were prepared in a total volume of $10 \mu \mathrm{l}$, containing $1 \mu \mathrm{l}$ cDNA, $0.6 \mu 1$ forward and reverse primer, $5 \mu$ l SYBR Green Supermix, $1 \mu \mathrm{l}$ plus solution (this solution is a component that increases the specificity and reliability of the PCR reaction) and $1.8 \mu \mathrm{l}$ RNase-free water. To amplify genomic DNA, PCR was performed under the following conditions: $95^{\circ} \mathrm{C}$ for $5 \mathrm{~min}$, followed by 40 cycles of $95^{\circ} \mathrm{C}$ for $10 \mathrm{sec}, 60^{\circ} \mathrm{C}$ for $10 \mathrm{sec}$, and $72^{\circ} \mathrm{C}$ for $10 \mathrm{sec}$. The quantification values were obtained from the quantification cycle $(\mathrm{Cq})$ number at which the increase in the signal was associated with an exponential growth of the PCR products. All samples were run in triplicate and repeated three times. RPS16 quantification was used as an internal control for normalization. Fold differences of mRNA levels over vehicle control were calculated by $2-^{\Delta \Delta \mathrm{Cq}}$ method (27).

Statistical analysis. Statistical analyses were carried out using the SPSS statistical software (version, 16.0; SPSS, Inc., Chicago, IL, USA). Numerical data of protein and mRNA assays were analyzed and compared by one-way analysis of variance (ANOVA) followed by a post hoc comparison test using the LSD (equal variances assumed) or Kruskal-Wallis (equal variances not assumed) method. Behavioral results with multiple comparisons were statistically analyzed by 
ANOVA for repeated measures. All values were presented as the means \pm standard deviation. $\mathrm{P}<0.05$ were considered to indicate a statistically significant difference.

\section{Results}

EA treatment reversed CCI-induced mechanical allodynia and thermal hyperalgesia. The MWT and TWL values were normalized to baseline (day 0 ) to determine the time course of CCI-induced mechanical allodynia and thermal hyperalgesia. There was no significant difference among the groups. In behavioral tests on day 3 post-CCI operation, MWT and TWL were significantly lower in the CCI group compared with the normal and sham-CCI groups $(\mathrm{P}<0.001)$. On day 7, the MWT was markedly reduced from $30.8 \pm 1.16$ to $10.7 \pm 1.28 \mathrm{~g}$, and TWL was reduced from $21.0 \pm 1.40$ to $10.3 \pm 0.54 \mathrm{sec}$ compared with the normal and sham-CCI groups $(\mathrm{P}<0.001)$. EA treatment markedly reduced the symptoms of neuropathic pain. As presented in Fig. 2A and B, EA significantly reversed the established mechanical allodynia and thermal hyperalgesia in the CCI model occurring on day 10. On day 14, the MWT and TWL of the CCI+EA group were markedly higher than those in the CCI group $(\mathrm{P}<0.001)$. Taken together, these results suggest that EA therapy effectively reduces the severity of pain induced by sciatic nerve injury.

EA upregulated $\mathrm{KCC} 2$ protein and mRNA levels in spinal cord. To explore the analgesic effect of EA, and prove the critical role of $\mathrm{KCC} 2$ for maintaining neuropathic pain, the authors next examined the protein and mRNA levels of $\mathrm{KCC} 2$ in the spinal cord following repeated EA treatments. Immunohistochemistry was performed in each group (Fig. 3A and B). Comparisons between the CCI and CCI+EA group revealed significant effect, $\mathrm{KCC} 2$ was evident in CCI+EA group relative to the $\mathrm{CCI}$ group. Furthermore, using western blotting, the authors examined the protein level of $\mathrm{KCC} 2$ in the L4-L6 spinal cord. CCI resulted in a significant reduction in the $\mathrm{KCC} 2$ protein levels compared to the sham-CCI group. The relative optical density value for $\mathrm{KCC} 2$ in the EA group was significantly higher than that in the $\mathrm{CCI}$ group $(\mathrm{P}<0.05$; Fig. 3C and D). RT-qPCR analysis confirmed the downregulation of $\mathrm{KCC} 2$ under chronic constriction at the sciatic nerve. However, following 7 days of EA treatment, $\mathrm{KCC} 2$ expression was significantly higher than it in the CCI group (Fig. 3E). Together, these results suggested that CCI may lead to a loss of KCC2 mRNA and protein, 2/100 Hz EA may increase KCC2 expression in the spinal cord of CCI rats, which, in turn may potentiate EA-induced analgesia in the spinal cord.

EA treatment increased spinal GABAA receptor $\gamma^{2}$ subunit expression in CCI rats. To evaluate the effect of EA on the GABAA receptor $\gamma 2$ subunit, immunohistochemistry, western blotting and RTq-PCR analyzed protein and mRNA levels. Direct analysis of GABAA receptor $\gamma 2$ subunit in the spinal cord was investigated using immunohistochemistry. As is demonstrated in Fig. 4A and B, EA significantly increased the expression of GABAA receptor $\gamma 2$ subunit $(\mathrm{P}<0.05)$. Western blotting produced similar results at the protein level $(\mathrm{P}<0.05$; Fig. 4C and D). Data from RT-qPCR revealed a prominent reduction in GABAA receptor $\gamma 2$ subunit mRNA in the spinal cord of CCI rats. However, EA significantly attenuated the CCI-induced downregulation of GABAA receptor $\gamma 2$
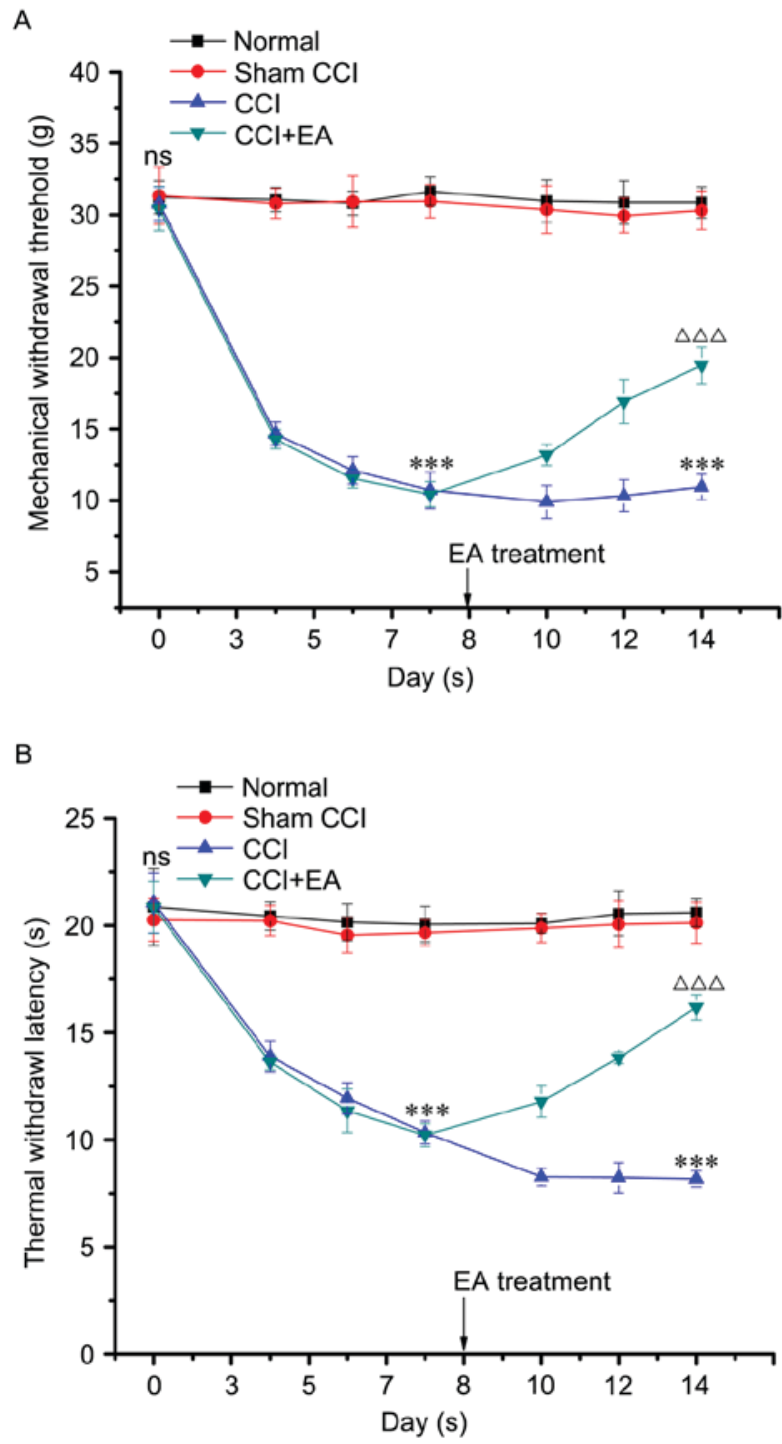

Figure 2. Treatment with EA resulted in a significant analgesic effect. (A) Mechanical allodynia. (B) Thermal hyperalgesia. The graph demonstrated the changes in withdrawal threshold from the baseline. The threshold was observed before (Pre) and following EA. MWT and TWL were significantly reduced from the baseline on day 3 following the CCI operation. MWT and TWL of rats treated with EA were significantly higher than the CCI group on the days indicated. ${ }^{* * *} \mathrm{P}<0.001$ vs. sham group; ${ }^{\Delta \Delta \Delta} \mathrm{P}<0.001$ vs. CCI group. CCI, chronic constriction injury; EA, electro-acupuncture.

subunit mRNA ( $\mathrm{P}<0.001$; Fig. 4E). These data suggested that CCI-induced NP inhibits the expression of GABAA receptor $\gamma 2$ subunit, thus indicating a potential role for GABAA receptor $\gamma 2$ subunit in mediating pain inhibition in response to CCI.

\section{Discussion}

The current study demonstrated that EA treatment has a beneficial effect on the pain threshold of rats undergoing CCI. The data indicated that the mechanical and thermal nociceptive thresholds maybe increased by EA on ST-36 and GB-34 acupoints in the CCI plus EA group. Furthermore, the authors examined the mRNA and protein levels of $\mathrm{KCC} 2$ and GABAA receptor $\gamma 2$ subunit in CCI rats and indicated that EA treatment significantly increased KCC 2 and GABAA receptor $\gamma 2$ subunit expression. 
A

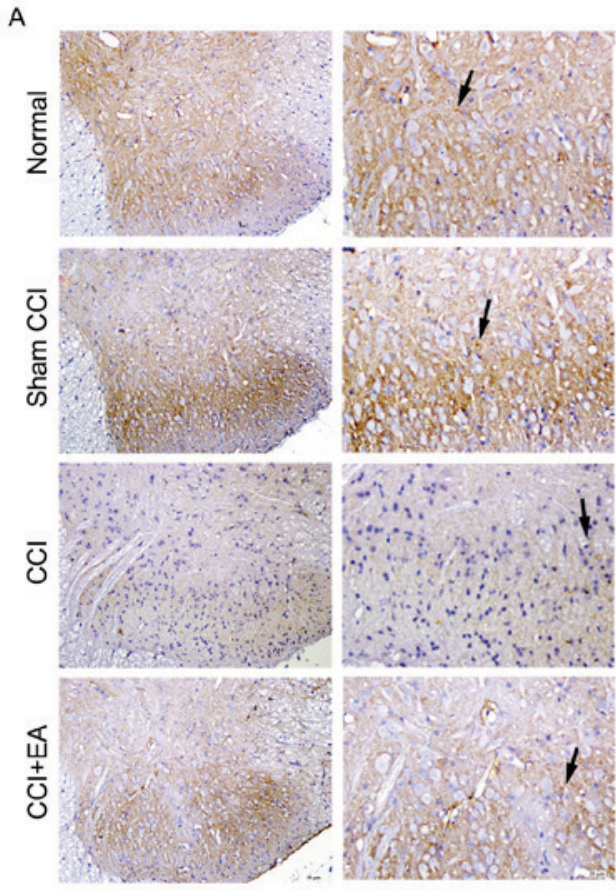

B

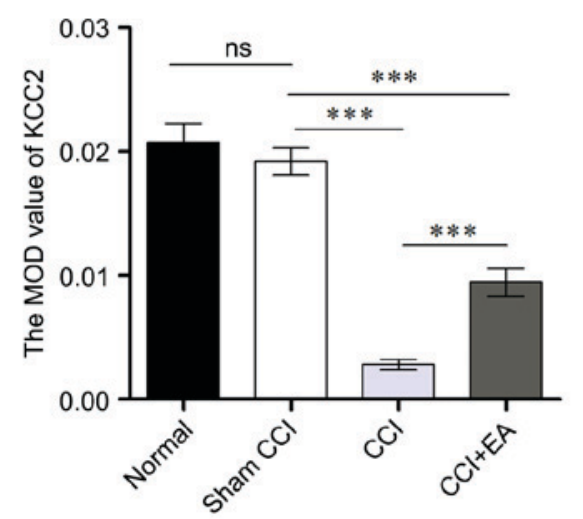

C
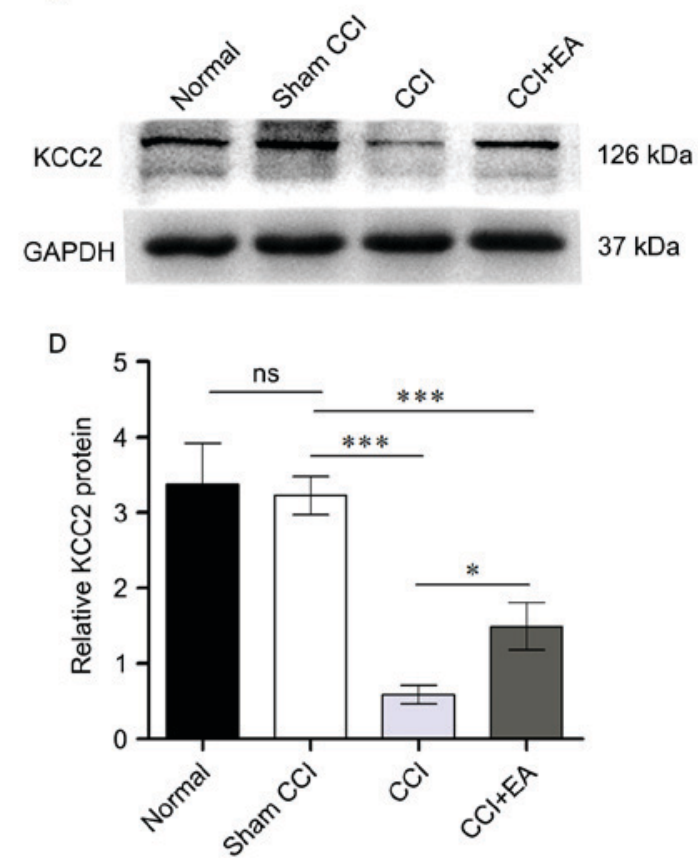

E

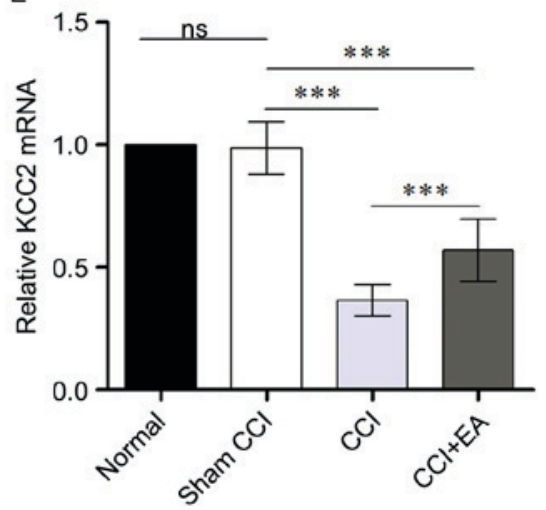

Figure 3. EA prevented KCC2 downregulation in CCI rats ( $=5$ ). (A) The KCC2 was stained with immunohistochemistry (The left side of the column: Scale bar, $50 \mu \mathrm{m}$; the right side of the column: Scale bar, $20 \mu \mathrm{m}$ ). (B) The bar graph indicated the quantitative KCC2 positive regions in four groups. (C) Western blot analysis of total KCC2 protein in L4-L6 demonstrated a marked reduction in CCI rats. GAPDH was used as the loading control. (D) Summary data from western blotting experiments. (E) Quantification of relative KCC2 mRNA levels in CCI rats. ${ }^{*} \mathrm{P}<0.05$, ${ }^{* * *} \mathrm{P}<0.001$ as indicated; ns, not significant. CCI, chronic constriction injury; EA, electro-acupuncture.

A great deal of evidence from diverse animal models of neuropathic pain has suggested that glia, particularly activated microglia, serve a critical role in the defense of the neural parenchyma (28). Following peripheral nerve injury, activated microglia will upregulate their surface expression of immunomodulatory proteins and release neurotransmitters and immunomodulators such as opioids, serotonin, noradrenaline and adenosine (29). Brain-derived neurotrophic factor (BDNF), which can be synthesized and released of microglia, has been reported to be crucial in the occurrence and development of NP (30). Most of the cellular behaviors of BDNF in the spinal cord are mediated by its high-affinity receptor tropomyosin-related kinase B (TrkB), and BDNF-TrkB has previously been shown to downregulate expression of the KCC2 $(6,30)$. It is reported that the BDNF-TrkB-KCC2 signaling pathway may contribute to NP not only during its occurrence but also during its maintenance (6). Based on these data, it was hypothesized that chronic pain may be correlated to KCC2 and its signal transduction pathway, the analgesic effect of EA may be associated with regulation of this pathway.

Accumulated evidence suggests that $\mathrm{KCC} 2$, the main $\mathrm{Cl}$ - transporter in spinal lamina I neurons, leads to decrease intracellular Cl- concentration (31). It serves a pivotal role in the inhibitory mechanisms that control neuronal excitation in the CNS (30). The downregulation of KCC2 has been implicated in various excitatory disorders, such as in epilepsy or in nerve injury resulting from various pathological conditions (32). As a result, GABAA receptors can be reduced which potentially shift the receptor-mediated responses from hyperpolarizing inhibition to depolarizing excitation $(32,33)$. The increased neuronal excitability may then sustain the enhanced (and exaggerated) communication between primary afferents and dorsal horn neurons to contribute to the early behavioral signs of pain (34).

The current study demonstrated that NP decreased the expression of KCC2 and GABAA receptor $\gamma 2$ subunit mRNA 
A

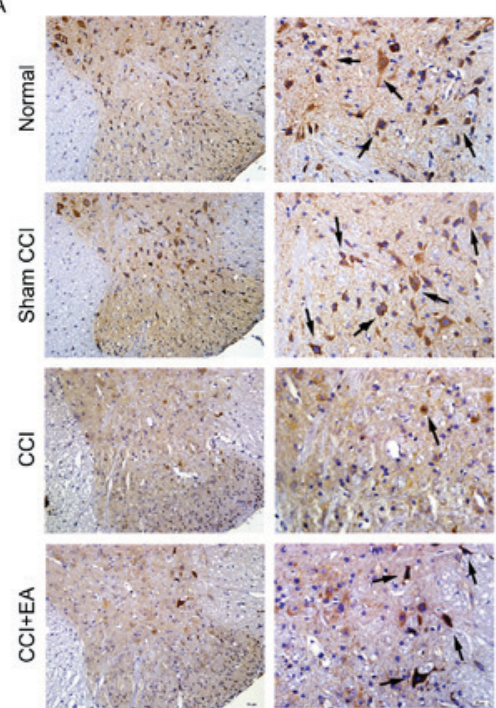

B

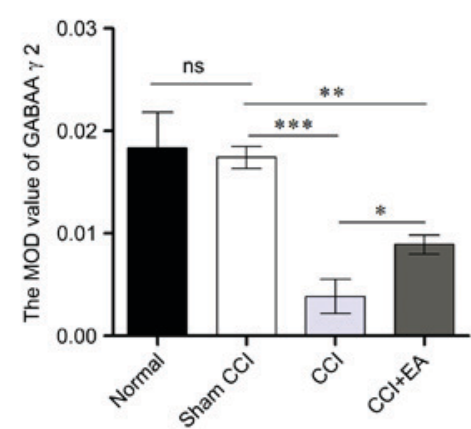

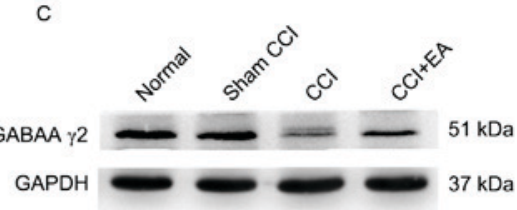

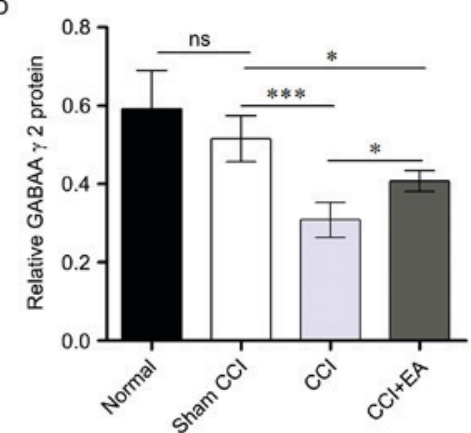

E

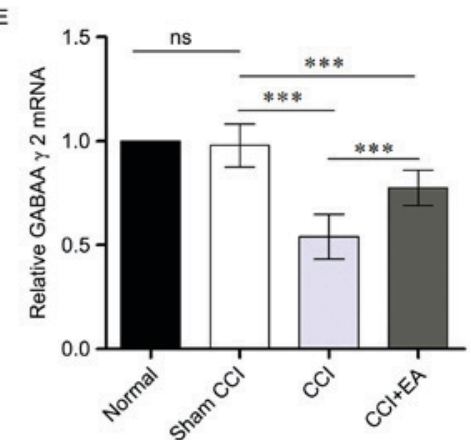

Figure 4. EA increased the expression of GABAA receptor $\gamma 2$ subunit $(\mathrm{n}=5)$. (A) The GABAA receptor $\gamma 2$ subunit was stained in an immunohistochemistry study (the left side of the column: Scale bar, $50 \mu \mathrm{m}$; the right side of the column: Scale bar, $20 \mu \mathrm{m}$ ). (B) The bar graph indicated the quantitative GABAA receptor $\gamma 2$ subunit positive regions in four groups. (C) Western blot analysis of GABAA receptor $\gamma 2$ subunit protein in L4-6 presented a marked reduction. GAPDH was used as the loading control. (D) Summary data from western blotting experiments. (E) Quantification of relative GABAA receptor $\gamma 2$ subunit mRNA levels in CCI rats. ${ }^{*} \mathrm{P}<0.05,{ }^{* * *} \mathrm{P}<0.001$ as indicated; ns, not significant. EA, electro-acupuncture; $\mathrm{CCI}$, chronic constriction injury;

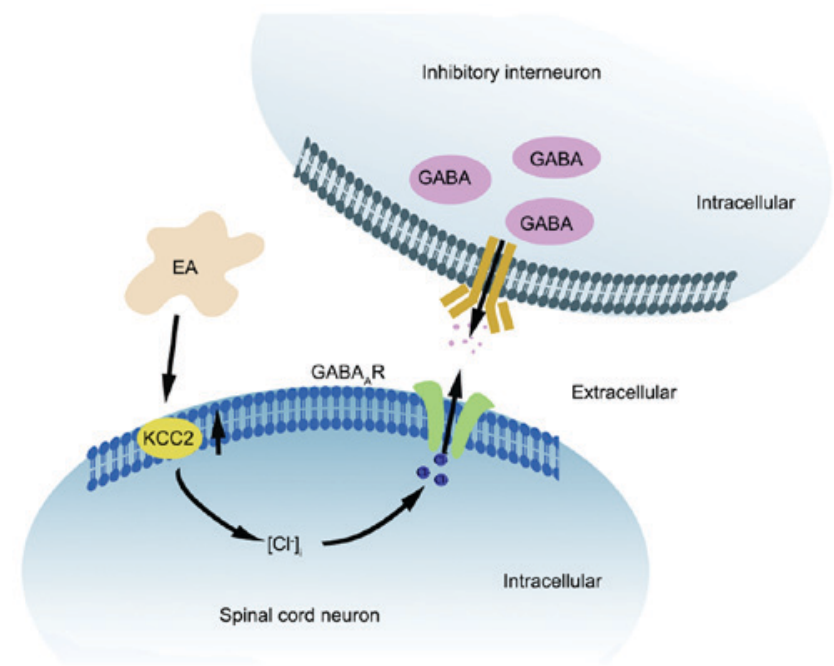

Figure 5. Outline of the current view on role of KCC2-GABAA receptor signaling pathway in chronic constriction injury induced neuropathic pain. Under normal conditions, KCC2 maintains a low concentration of intracellular Cl-. Peripheral nerve injury causes a differential change of $\mathrm{KCC} 2$ expression and function consequently increases intracellular Cl- concentration and hinders GABAA receptor-mediated inhibition of neuronal excitability. The analgesic effects of EA may be achieved via regulation of the $\mathrm{KCC} 2-\mathrm{GABAA}$ receptor signaling pathway in the central sensory. EA, electro-acupuncture. and protein in CCI rats and this occurred concomitantly with a marked increase in allodynia. However, EA significantly relieved mechanical allodynia and thermal hyperalgesia and increased the expression of KCC2 and GABAA receptor $\gamma 2$ subunit.

EA has been established as a viable therapeutic intervention to alleviate chronic pain and has shown positive effects in the clinic, but the biological basis of its action remains unknown (35). Several studies have demonstrated that EA has an immunomodulatory effect (36). Zhang Wang and McAlonan (37) suggested that acupuncture stimulation can release pain killers and CNS mediators, including endorphin, morphine and GABA. GABA has been implicated not only in peripheral responses but also in interfering with pain impulse input in the CNS. In the present study, EA prevented decreased levels of KCC2 and GABAA in the central sensory pathways and reduced NP following peripheral nerve injury. Based on these findings, the authors suspect that the mechanical allodynia and thermal hyperalgesia mediated by KCC2-GABAA receptor signaling pathway is attenuated by EA via its immunomodulatory effects in the spinal cord (Fig. 5). To further characterize the analgesic effect of EA, pharmacology, electrophysiological techniques and other methods will be conducted in the future. 
In conclusion, these data suggested that peripheral nerve injury caused a reduction in KCC2 and GABAA receptor $\gamma 2$ subunit expression in the spinal cord and that the upregulation of KCC 2 and GABAA receptor $\gamma 2$ subunit may account for the analgesic effect of EA. Overall, the results of the present initial study provide a promising foundation for future research on the impact of EA on NP.

\section{Acknowledgements}

Not applicable.

\section{Funding}

The current study was supported by the Natural Science Foundation of Zhejiang Province (grant no. LY16H270016) and the Foundation of Wenzhou Scientific and Technological Bureau Project (grant no. Y20140221).

\section{Availability of data and materials}

The analyzed data sets generated during the study are available from the corresponding author on reasonable request.

\section{Authors' contributions}

SL, WT, SJ and BL conceived and designed the experiments. CJ, WC and XJ performed the experiments. XQ, GY and QH analyzed the data and prepared the figures. WT and SL wrote the paper. BL and SJ contributed to the modification of the manuscript. All authors read and approved the final manuscript.

\section{Ethics approval and consent to participate}

All experiments were maintained and cared for according to the guidelines of the National Institutes of Health Guide for the Care and Use of Laboratory Animals (NIH Publications No. 80-23, revised 1978). The experimental protocol was approved by the Institutional Animal Care and Use Committee of Wenzhou Medical University (Wenzhou, China).

\section{Consent for publication}

Not applicable.

\section{Competing interests}

The authors declare they have no competing interests.

\section{References}

1. Amit $\mathrm{Z}$ and Galina ZH: Stress-induced analgesia: Adaptive pain suppression. Physiol Rev 66: 1091-1120, 1986.

2. Fox A, Kesingland A, Gentry C, McNair K, Patel S, Urban L and James I: The role of central and peripheral Cannabinoid1 receptors in the antihyperalgesic activity of cannabinoids in a model of neuropathic pain. Pain 92: 91-100, 2001.

3. Woolf CJ and Mannion RJ: Neuropathic pain: Aetiology, symptoms, mechanisms, and management. Lancet 353: 1959-1964, 1999.
4. Miletic G and Miletic V: Loose ligation of the sciatic nerve is associated with TrkB receptor-dependent decreases in KCC2 protein levels in the ipsilateral spinal dorsal horn. Pain 137: 532-539, 2008.

5. Zhang W, Liu LY and Xu TL: Reduced potassium-chloride co-transporter expression in spinal cord dorsal horn neurons contributes to inflammatory pain hypersensitivity in rats. Neuroscience 152: 502-510, 2008.

6. Coull JA, Boudreau D, Bachand K, Prescott SA, Nault F, Sík A, De Koninck P and De Koninck Y: Trans-synaptic shift in anion gradient in spinal lamina I neurons as a mechanism of neuropathic pain. Nature 424: 938-942, 2003.

7. Delpire E: Cation-chloride cotransporters in neuronal communication. News Physiol Sci 15: 309-312, 2000.

8. Medina I, Friedel P, Rivera C, Kahle KT, Kourdougli N, Uvarov P and Pellegrino C: Current view on the functional regulation of the neuronal $\mathrm{K}(+)-\mathrm{Cl}(-)$ cotransporter KCC2. Front Cell Neurosci 8: 27, 2014.

9. Acton BA, Mahadevan V, Mercado A, Uvarov P, Ding Y, Pressey J, Airaksinen MS, Mount DB and Woodin MA: Hyperpolarizing GABAergic transmission requires the $\mathrm{KCC} 2 \mathrm{C}$-terminal ISO domain. J Neurosci 32: 8746-8751, 2012.

10. Price TJ, Cervero F and de Koninck Y: Role of cation-chloride-cotransporters (CCC) in pain and hyperalgesia. Curr Top Med Chem 5: 547-555, 2005.

11. Price TJ, Cervero F, Gold MS, Hammond DL and Prescott SA: Chloride regulation in the pain pathway. Brain Res Rev 60: 149-170, 2009.

12. Bowery NG, Hudson AL and Price GW: GABAA and GABAB receptor site distribution in the rat central nervous system. Neuroscience 20: 365-383, 1987.

13. Malherbe P, Sigel E, Baur R, Persohn E, Richards JG and Mohler H: Functional characteristics and sites of gene expression of the alpha 1, beta 1, gamma 2-isoform of the rat GABAA receptor. J Neurosci 10: 2330-2337, 1990.

14. Barnard EA, Skolnick P, Olsen RW, Mohler H, Sieghart W, Biggio G, Braestrup C, Bateson AN and Langer SZ: International union of pharmacology. XV. Subtypes of gamma-aminobutyric acidA receptors: Classification on the basis of subunit structure and receptor function. Pharmacol Rev 50: 291-313, 1998.

15. Bonnert TP, McKernan RM, Farrar S, le Bourdellès B, Heavens RP, Smith DW, Hewson L, Rigby MR, Sirinathsinghji DJ, Brown N, et al: theta, a novel gamma-aminobutyric acid type A receptor subunit. Proc Natl Acad Sci USA 96: 9891-9896, 1999.

16. Sieghart W and Sperk G: Subunit composition, distribution and function of GABA(A) receptor subtypes. Curr Top Med Chem 2: 795-816, 2002.

17. Araki T, Sato M, Kiyama H, Manabe Y and Tohyama M: Localization of GABAA-receptor gamma 2-subunit mRNA-containing neurons in the rat central nervous system. Neuroscience 47: 45-61, 1992.

18. Kelly RB: Acupuncture for pain. Am Fam Physician 80: 481-484, 2009.

19. Ulett GA, Han S and Han JS: Electroacupuncture: Mechanisms and clinical application. Biol Psychiatry 44: 129-138, 1998.

20. Tu W, Wang W, Xi H, He R, Gao L and Jiang S: Regulation of neurotrophin-3 and interleukin-1 $\beta$ and inhibition of spinal glial activation contribute to the analgesic effect of electroacupuncture in chronic neuropathic pain states of rats. Evid Based Complement Alternat Med 2015: 642081, 2015.

21. Wang WS, Tu WZ, Cheng RD, He R, Ruan LH, Zhang L, Gong YS, Fan XF, Hu J, Cheng B, et al: Electroacupuncture and A-317491 depress the transmission of pain on primary afferent mediated by the $\mathrm{P} 2 \mathrm{X} 3$ receptor in rats with chronic neuropathic pain states. J Neurosci Res 92: 1703-1713, 2014.

22. Tu WZ, Cheng RD, Cheng B, Lu J, Cao F, Lin HY, Jiang YX, Wang JZ, Chen H and Jiang SH: Analgesic effect of electroacupuncture on chronic neuropathic pain mediated by $\mathrm{P} 2 \mathrm{X} 3$ receptors in rat dorsal root ganglion neurons. Neurochem Int 60: 379-386, 2012.

23. Lee CH, Kim DK, Yook TH, Sasaki M and Kitamura N: Effectiveness of electroacupuncture at Zusanli (ST36) on the immunohistochemical density of enteroendocrine cells related to gastrointestinal function. J Acupunct Meridian Stud 5: 63-71, 2012.

24. Chen SP, Kan Y, Zhang JL, Wang JY, Gao YH, Qiao LN, Feng XM, Yan YX and Liu JL: Involvement of hippocampal acetylcholinergic receptors in electroacupuncture analgesia in neuropathic pain rats. Behav Brain Funct 12: 13, 2016. 
25. Wang Y, Zhang Y, Wang W, Cao Y and Han JS: Effects of synchronous or asynchronous electroacupuncture stimulation with low versus high frequency on spinal opioid release and tail flick nociception. Exp Neurol 192: 156-162, 2005.

26. Silva JR, Silva ML and Prado WA: Analgesia induced by 2- or $100-\mathrm{Hz}$ electroacupuncture in the rat tail-flick test depends on the activation of different descending pain inhibitory mechanisms. J Pain 12: 51-60, 2011.

27. Livak KJ and Schmittgen TD: Analysis of relative gene expression data using real-time quantitative PCR and the 2(-Delta Delta C(T)) method. Methods 25: 402-408, 2001.

28. Cao $\mathrm{H}$ and Zhang YQ: Spinal glial activation contributes to pathological pain states. Neurosci Biobehav Rev 32: 972-983, 2008.

29. Suzuki T, Hide I, Ido K, Kohsaka S, Inoue K and Nakata Y: Production and release of neuroprotective tumor necrosis factor by P2X7 receptor-activated microglia. J Neurosci 24: 1-7, 2004.

30. Coull JA, Beggs S, Boudreau D, Boivin D, Tsuda M, Inoue K, Gravel C, Salter MW and De Koninck Y: BDNF from microglia causes the shift in neuronal anion gradient underlying neuropathic pain. Nature 438: 1017-1021, 2005.

31. Kahle KT, Staley KJ, Nahed BV, Gamba G, Hebert SC, Lifton RP and Mount DB: Roles of the cation-chloride cotransporters in neurological disease. Nat Clin Pract Neurol 4: 490-503, 2008.
32. Zhang D, Gopalakrishnan SM, Freiberg G and Surowy CS: A thallium transport FLIPR-based assay for the identification of KCC2-positive modulators. J Biomol Screen 15: 177-184, 2010.

33. De Koninck Y: Altered chloride homeostasis in neurological disorders: A new target. Curr Opin Pharmacol 7: 93-99, 2007.

34. Nomura H, Sakai A, Nagano M, Umino M and Suzuki H: Expression changes of cation chloride cotransporters in the rat spinal cord following intraplantar formalin. Neurosci Res 56: 435-440, 2006.

35. Berman BM,Langevin HM, Witt CM and Dubner R: Acupuncture for chronic low back pain. N Engl J Med 363: 454-461, 2010

36. Ouyang BS, Gao J, Che JL, Zhang Y, Li J, Yang HZ, Hu TY, Yang M, Wu YJ and Ji LL: Effect of electro-acupuncture on tumor necrosis factor- $\alpha$ and vascular endothelial growth factor in peripheral blood and joint synovia of patients with rheumatoid arthritis. Chin J Integr Med 17: 505-509, 2011.

37. Zhang ZJ, Wang XM and McAlonan GM: Neural acupuncture unit: A new concept for interpreting effects and mechanisms of acupuncture. Evid Based Complement Alternat Med 2012: 429412, 2012.

(i) (1) This work is licensed under a Creative Commons

c) AY NG Attribution-NonCommercial-NoDerivatives 4.0 International (CC BY-NC-ND 4.0) License. 